\title{
LA PRODUCCIÓN ORAL EN LENGUAS EXTRANJERAS MÁS ALLÁ DEL AULA CON EL FORO DE VOZ VOXOPOP
}

\author{
Fátima Faya Cerqueiro/Universidad de Castilla-La Mancha*
}

\begin{abstract}
RESUMEN: Este artículo expone el uso de una herramienta para la producción oral de voz en el contexto de enseñanza de una lengua extranjera. Los docentes de lenguas extranjeras y segundas lenguas solemos encontrarnos ante grupos más numerosos de lo deseado, en los que nos resulta casi imposible asegurar la intervención de todo el alumnado de un modo equitativo. El foro de voz VOXOPOP supone un gran avance como herramienta docente, ya que nos permite incrementar el tiempo destinado a la producción oral del alumno, mediante la asignación de tareas orales como trabajo de revisión, de refuerzo o apoyo. Esta herramienta se ha probado en un grupo de alumnos de lengua inglesa de nivel B1, que estudian un grado universitario de carácter no lingüístico. VOXOPOP ha mostrado una gran utilidad para llevar a cabo tareas que plantean discusiones sobre temas específicos de escasa complejidad.
\end{abstract}

PALABRAS CLAVE: Foro de voz. VOXOPOP. Producción oral. Enseñanza de una lengua extranjera.

RESUMO: Este artigo expõe o uso de uma ferramenta para a produção oral de voz no contexto de ensino de uma língua estrangeira. Os docentes de línguas estrangeiras e segundas línguas habitualmente se encontram diante de turmas mais numerosas do que o desejado, e resulta quase impossível assegurar a intervenção de todos os alunos de um modo equitativo. O foro de voz VOXOPOP supõe um grande avanço como ferramenta docente, já que permite incrementar o tempo destinado à produção oral do aluno, mediante a atribuição de tarefas orais como trabalho de revisão, de reforço ou de apoio. Esta ferramenta foi testada numa turma de alunos de língua inglesa de nível B1, que fazem um curso universitário de caráter não-linguístico. VOXOPOP mostrou uma grande utilidade para levar a cabo tarefas que propõem discussões sobre temas específicos de escassa complexidade.

PALAVRAS-CHAVE: Foro de voz. VOXOPOP. Produção oral. Ensino de uma língua estrangeira.

\section{INTRODUCCIÓN}

Son numerosos los estudios que justifican el uso de distintas herramientas digitales para favorecer la mejora de destrezas lingüísticas por parte de estudiantes de segundas lenguas o de

* Agradezco las sugerencias de los revisores anónimos a una versión previa de este artículo. Asimismo, se reconoce la financiación del Fondo Europeo de Desarrollo Regional, del Ministerio de Economía y Competitividad (beca FFI2011-26693-C02-01), así como de la Xunta de Galicia (becas CN2011-011 y CN2012/012). 
lenguas extranjeras. En este contexto, las destrezas relativas a comprensión escrita y auditiva se han visto favorecidas de inmediato, debido a la facilidad que supone alojar archivos en webs, blogs o campus virtuales para que el alumno los pueda descargar y trabajar con ellos de un modo totalmente autónomo. GÓMEZ MARTÍNEZ (2010) ofrece una muestra del amplio abanico de posibilidades que existen para trabajar la comprensión oral haciendo uso de las nuevas tecnologías. Sin embargo, con el incremento y la mejora de las nuevas tecnologías cada vez son más las ventajas que nos ofrecen el software libre y las plataformas virtuales para mejorar destrezas que hasta ahora se limitaban a la presencialidad del aula. Una experiencia interesante en este sentido está relacionada con el uso de podcasts como material de apoyo o para proporcionar feedback individualizado al alumno sobre su trabajo (EDIRISINGHA; HAWKRIDGE; FOTHERGILL, 2010). Antes del actual desarrollo de las nuevas tecnologías, algunos autores ya señalaban la importancia del diálogo entre profesor y alumno en la educación a distancia, sobre todo cuando se trata de adultos en contextos especializados (EVANS; NATION, 1989).

En cuanto al perfeccionamiento de las destrezas que se refieren a la expresión y a la interacción oral, RICHARDSON (2004) menciona el potencial de las nuevas tecnologías en la mejora de la alfabetización en sentido amplio, y de la expresión oral en la lengua materna del alumno, basándose en experiencias llevadas a cabo en cuatro países. Asimismo, BRACEY (2005) hace referencia a experiencias concretas del uso de las nuevas tecnologías en distintos países, ligadas en su caso a la enseñanza de lenguas extranjeras. Este autor indica los beneficios en distintas destrezas lingüísticas y da como ejemplo la mejora de la expresión oral en alumnos de enseñanza primaria. La práctica de la producción oral en una lengua extranjera o segunda lengua resulta determinante no sólo para mejorar la expresión e interacción oral del alumno, sino también para consolidar los contenidos que va adquiriendo. De igual modo, a través de la expresión oral el estudiante desarrollará estrategias para resolver las dificultades de tipo lingüístico que surgen al comunicarse (ARNAIZ; PEÑATE, 2004). En cuanto a la producción oral, algunos autores han puesto de manifiesto la utilidad de ambientes virtuales para el desarrollo de esta destreza a través de chats escritos (VETTER; CHANIER, 2006). A pesar de usar distintos medios, fónico y gráfico respectivamente, tanto la producción oral en el aula como el uso de chats pueden encuadrarse en el denominado 'lenguaje de la inmediatez comunicativa' (ÖSTERREICHER 1997; KOCH 1999), por lo que la expresión escrita supone una gran ayuda para la expresión oral del alumno.

La experiencia que se describe en el presente trabajo está basada en la utilización de un foro de voz y su potencialidad para mejorar la producción oral del alumnado. El foro de voz VOXOPOP es una herramienta gratuita, accesible a través de la página web www.voxopop.com, creada y desarrollada por Dean Worth, inicialmente bajo el nombre Chinswing, y con la nueva denominación “Voxopop” a partir de 2009, definida como una “voice-based e-learning tool”, por lo que desde su origen está planteada con una finalidad docente. Esto se pone de manifiesto en el uso que han hecho otros autores de la herramienta con fines didácticos o de carácter evaluativo (SHIPLEY; MUILENBURG, 2012). Por su parte, FARRAND (2011, p. 24-25) hace algunas sugerencias de actividades que se pueden realizar con este foro de voz en la enseñanza-aprendizaje de una lengua extranjera. Un sistema similar a VOXOPOP es el que ofrece VoiceThread ${ }^{i}$, una plataforma similar, que funciona mediante el pago de una licencia y permite además subir archivos y comentarlos mediante texto y voz. 


\section{VOXOPOP}

En la página principal de VOXOPOP encontramos la posibilidad de crear un grupo al que tengan acceso nuestros alumnos, en el cual pueden dejar plasmadas sus intervenciones de forma estrictamente oral. La página principal, cuya interfaz se muestra en la Figura 1, nos permite acceder a grupos sin acceso restringido, en los que resulta sencillo ver cómo están organizados, cómo se visualizan las intervenciones o incluso comprobar la calidad del sonido.

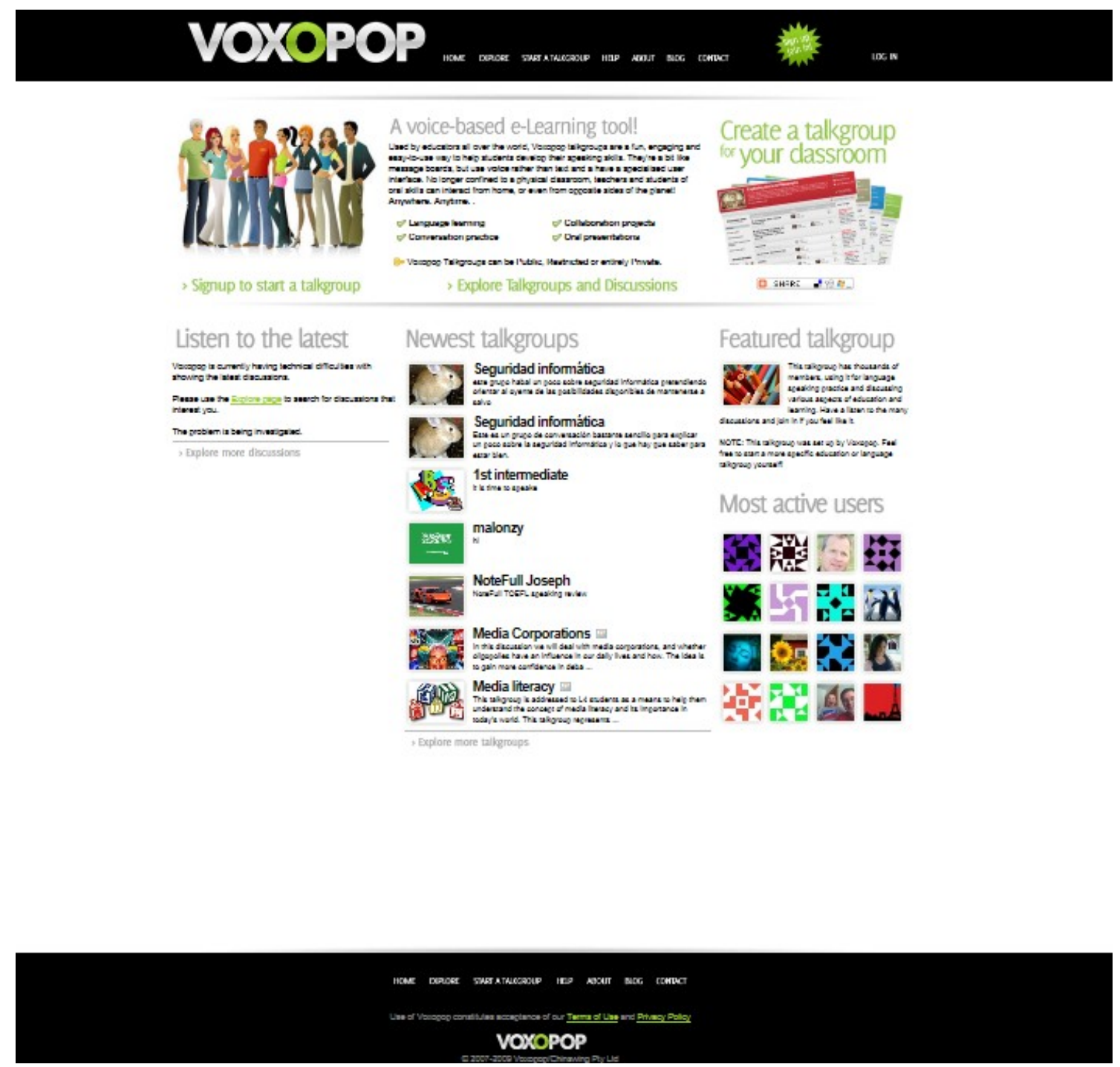

Figura 1: Página inicial de VOXOPOP

Además, podemos crear nuestro propio grupo a través de la página principal, clicando en "Create a talkgroup for your classroom". Al crear nuestro grupo lo haremos en calidad de profesor, para asegurarnos el acceso a las modificaciones y propuestas del foro. Nos dan la opción de subir una imagen a modo de avatar, algo que suele resultar atractivo para los alumnos, y de igual modo podemos crear una imagen que describa nuestro grupo. Las opciones que dan programas como Impress o Draw, ambos de OpenOffice ${ }^{\mathrm{ii}}$, pueden facilitarnos la creación de un logo con el que 
nuestros estudiantes se sientan identificados.

Una vez que estamos registrados ya tenemos acceso a la herramienta que nos permite grabar una presentación oral, algo que será muy conveniente para animar a nuestros alumnos a hacer lo mismo. Podremos crear ya nuestro "talkgroup" y empezar a enviar las invitaciones correspondientes a nuestros estudiantes. Debemos fijarnos en que las restricciones contra el correo basura limitan el envío de invitaciones a grupos de 20 direcciones de e-mail como máximo, por lo que el proceso puede ser largo cuando las cifras de nuestras clases son superiores. Los alumnos recibirán las invitaciones en su e-mail, junto con las instrucciones que les hayamos dado. Cuando hayan sido invitados, podrán registrarse como estudiantes y tener acceso al foro, aunque más restringido que el del profesor-administrador, como es obvio.

\section{CONTEXTO}

El uso de VOXOPOP se comprobó en varios grupos de alumnado universitario, correspondiente a la Facultad de Ciencias del Deporte en la Universidad de Castilla-La Mancha. Los estudiantes cursan una asignatura de inglés de nivel B1, según el Marco Común Europeo de Referencia para las lenguas (MCER) (2002), en la que hay un marcado enfoque hacia las lenguas para fines específicos, debido al grado en el que se imparte y a los intereses del grupo. Esta orientación como lengua de especialidad afecta sobre todo la docencia del léxico, que incluye vocabulario deportivo, relacionado con la salud, el cuerpo humano y con otros aspectos académicos y profesionales. En menor medida, también se extiende a otras áreas del lenguaje, como la gramática o la pragmática. Sin embargo, el aprendizaje de estrategias de producción oral, la fonética o la pronunciación no se ven afectadas por el sesgo específico del curso, y siguen las directrices aconsejadas en el MCER (2002). El MCER (2002) propone una división de destrezas lingüísticas desmarcándose de divisiones más tradicionales, incluyendo no sólo expresión escrita, comprensión auditiva y comprensión escrita, sino también estableciendo una diferencia entre expresión e interacción en las destrezas orales. Mientras que la interacción oral requiere la presencia de un interlocutor, la producción monologada puede realizarse de forma autónoma. Dado el gran número de alumnos en el curso mencionado, se creyó conveniente probar esta herramienta para diseñar actividades que el estudiante pudiese llevar a cabo desde su casa para mejorar la producción oral.

\section{FUNCIONAMIENTO Y METODOLOGÍA}

Es necesario indicar que VOXOPOP ofrece la posibilidad de que los grupos sean accesibles para otros usuarios o que estén limitados a aquellos que el profesor-administrador decide. A menos que nuestros estudiantes tengan un nivel muy avanzado, es preferible que el acceso esté restringido, ya que los alumnos no sentirán que cualquier persona puede juzgarles, ya que sólo les escucharán sus compañeros de aula, con los que ya existe confianza y están acostumbrados a escucharse y corregirse unos a otros en la enseñanza presencial.

Además de la temática a discutir, podemos crear unas reglas propias para nuestro grupo, como por ejemplo prohibir que lean y evitar así que reproduzcan textos previamente escritos, o bien 
pedirles que escuchen todas las intervenciones previas para no repetirse o para mostrar su acuerdo o desacuerdo con opiniones previas. En la Figura 2 se puede comprobar la visión que tenemos de la tarea propuesta cuando van interviniendo varios alumnos. La participación de cada miembro del grupo queda registrada por orden de intervención, si bien también pueden responder a un compañero o abrir un nuevo hilo, como ocurre en los foros escritos tradicionales.

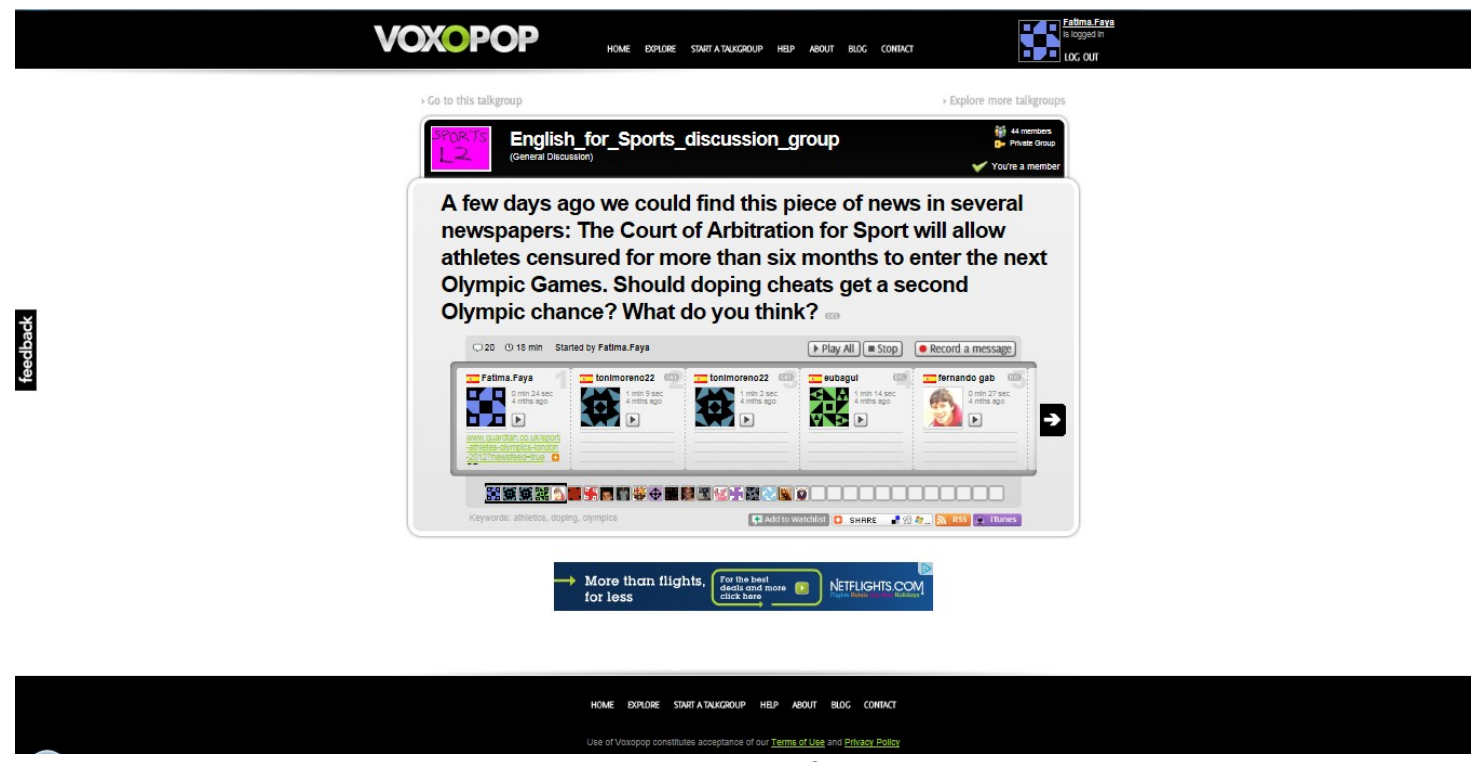

Figura 2: Formulación de tareas

A diferencia de otro tipo de trabajos que tengan que realizar para clase, los alumnos suelen intentar hacer este tipo de tareas cuanto antes para evitar tener que escuchar demasiadas intervenciones. Podemos observar cómo se organizan las intervenciones en la Figura 3, de tal modo que en una primera columna aparecen los títulos que los alumnos han dado a sus intervenciones. A continuación, los alumnos que han propuesto ese tema o que han respondido al mismo hilo. La última columna recoge el tiempo total invertido en un tema concreto, el cual podría equipararse al número de páginas que alcanza un hilo de debate en un foro escrito. 


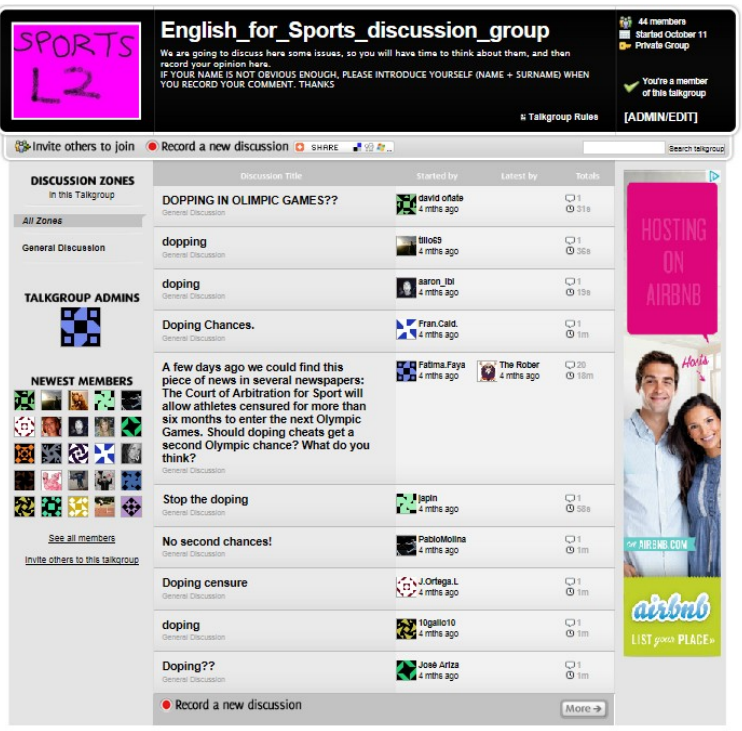

Figura 3: Intervenciones

En cualquier momento podemos comprobar qué alumnos se han registrado accediendo a la pestaña de miembros de nuestro grupo, como se puede ver en la Figura 4, en la que cada imagen representa a un miembro del grupo. También podemos enviar invitaciones de nuevo a la misma dirección de e-mail si la cuenta de correo se la ha detectado como spam, aunque después de la experiencia, se ha comprobado que esto ocurre en muy pocos casos.

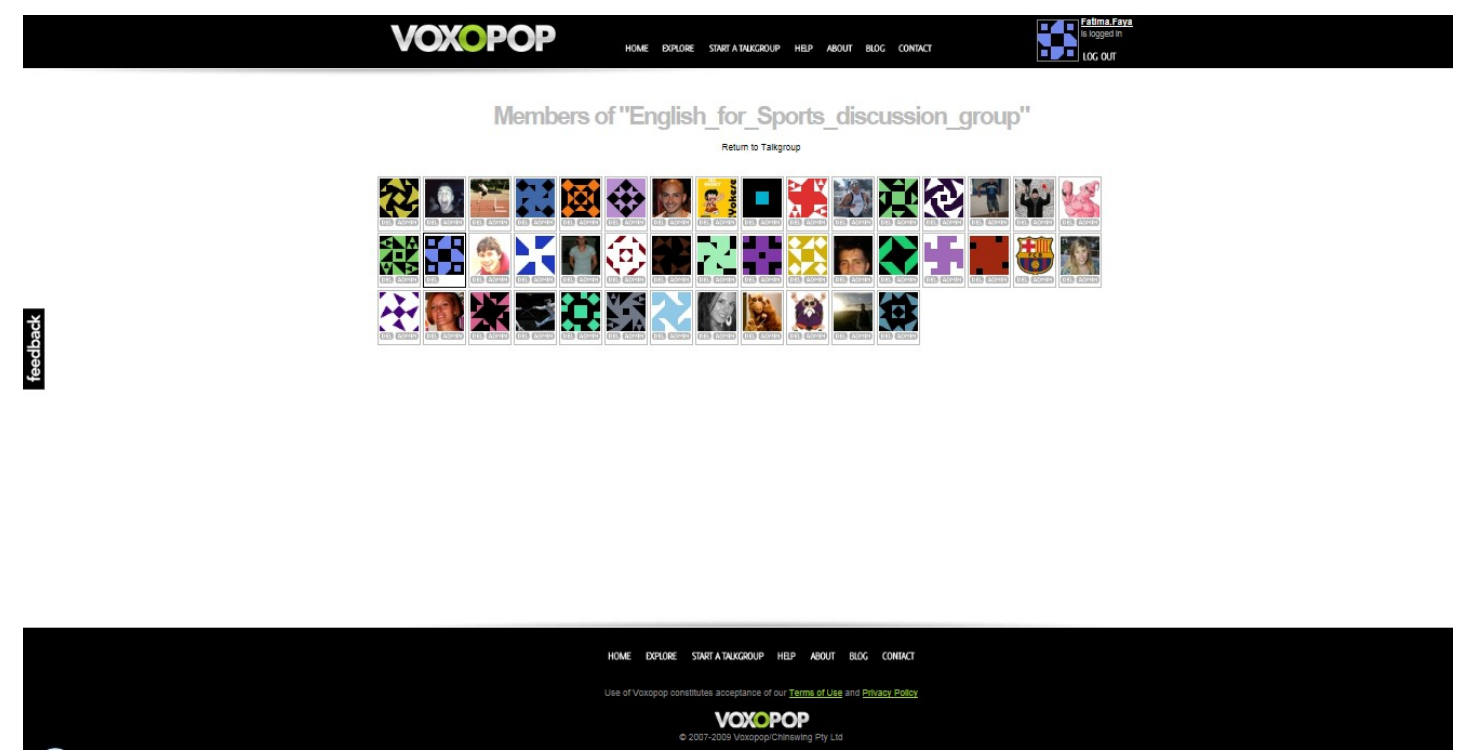

Figura 4: Participantes

Los estudiantes pueden hacer pruebas de su intervención y escucharlas tantas veces como deseen, hasta que por fin deciden subir su grabación como definitiva. Los alumnos echan de menos no poder subir un archivo con una grabación previamente almacenada en su equipo, aunque la herramienta que ofrece VOXOPOP es fácil de usar, manifiesta una gran fiabilidad e incluye otras 
opciones como insertar un título a la intervención o asignar palabras clave que la describan. Se muestra la interfaz de la herramienta de grabación en la Figura 5. Los participantes sólo necesitarán utilizar un micrófono, que normalmente ya está integrado en la mayoría de ordenadores portátiles.

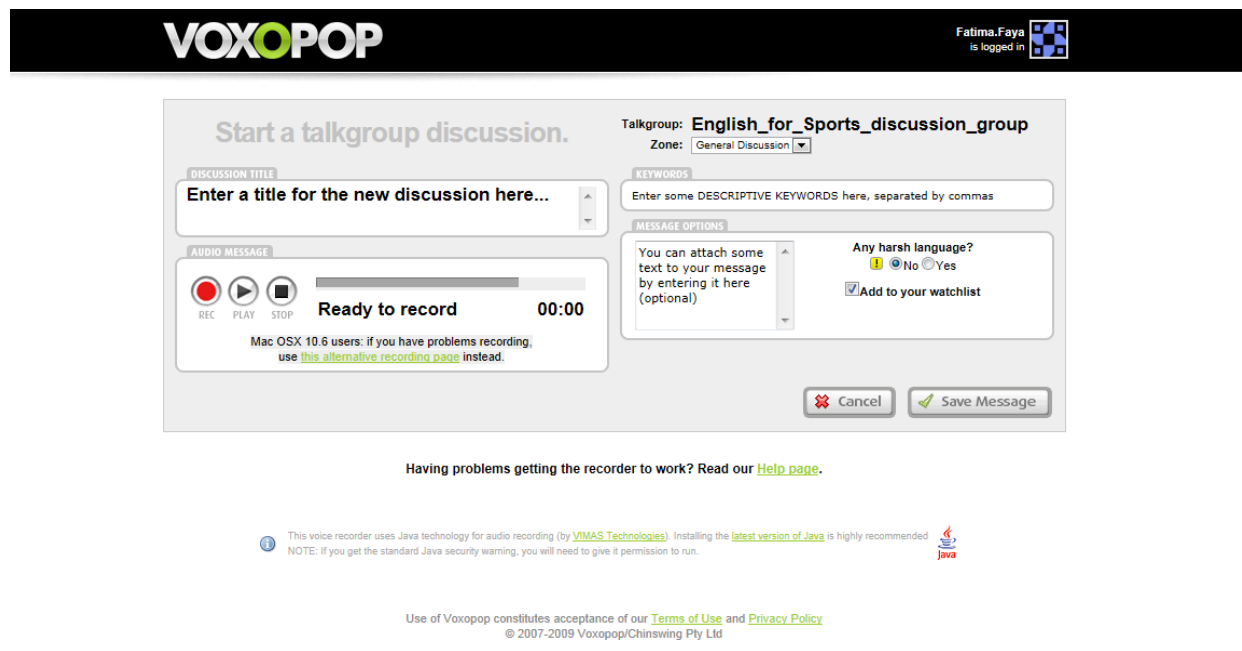

Figura 5: Herramienta de grabación

La tarea que se les propone suele tener un cierto carácter de debate, para provocar que las opiniones sean diferentes y que entren en acuerdo o desacuerdo con otros compañeros. En la Figura 2 veíamos una de las temáticas propuestas, acerca del dopaje, que siempre da lugar a variadas interpretaciones. El plazo para llevar a cabo la tarea suele ser de una semana, lo que facilita que el alumno tenga tiempo a buscar información y poder así argumentar mejor su opinión.

En niveles muy básicos, como los correspondientes a A1 e incluso a A2 según el MCER (2002), el uso de esta herramienta podría enfocarse hacia tareas de pronunciación, como identificación o reproducción de sonidos o palabras. En cualquier caso, las tareas requerirán la realización de textos breves, ya que la producción monologada con intervenciones largas resultará mucho más complicada para estos alumnos. En este sentido, puede aprovecharse para poner en práctica aspectos pragmáticos, como es el caso de algunos actos de habla que siguen patrones lingüísticos muy definidos, por ejemplo presentaciones, felicitaciones o agradecimientos, que no requieren la respuesta inmediata de un interlocutor.

\section{INCORPORACIÓN DE OTRAS HERRAMIENTAS}

Las sugerencias que hacemos a los alumnos de cómo llevar a cabo la tarea pueden mejorar su productividad. Un buen consejo suele ser que cuando quieran introducir vocabulario difícil en su intervención tendrán que asegurarse una correcta pronunciación. El alumno medio tiende a ser reacio a usar el diccionario, pero las ventajas de los diccionarios en línea pueden romper esa barrera. De esta manera, los archivos de voz que muchos diccionarios adjuntan a las entradas, 
suelen provocar que el alumno escuche y repita varias veces la palabra que busca, por lo que potenciamos su producción oral y escrita, y la consiguiente asimilación de léxico. Uno de los diccionarios que ofrecen esta posibilidad es http://www.thefreedictionary.com ${ }^{\mathrm{iii}}$, que incluye tanto pronunciación británica como americana. Otra herramienta interesante para el mismo fin, es una aplicación gratuita de Google Chrome, disponible para usuarios registrados a través de Chrome Web Store, denominada "How Do You Say"iv, la cual permite al usuario que al hacer clic en una palabra en inglés cuando se navega por la web, escuche su pronunciación correcta. De esta forma, mientras el alumno lee un artículo de prensa en lengua inglesa puede visualizar nuevo vocabulario en su contexto y también escucharlo.

\section{VENTAJAS E INCONVENIENTES}

Un problema que nos encontramos con el uso del foro de voz es la tentación que tiene el alumno de leer un texto previamente elaborado. Aunque es algo difícil de evitar, lo cierto es que se nota mucho cuando lo hacen, por lo que podemos no evaluar esa intervención y pedirle que haga una nueva, cumpliendo con las normas del foro. La adecuación de las tareas al nivel del alumno puede limitar que esto ocurra, ya que se comprueba que a medida que las tareas van aumentando su complejidad, los alumnos con más problemas de aprendizaje son también los que recurren más a menudo a leer un texto previamente elaborado.

Sin embargo, las ventajas que esta herramienta presenta son muchas más que los inconvenientes que nos pueda plantear, ya que estamos superando los límites físicos, espaciales y temporales del aula. Nos aseguramos que el alumno hable el tiempo estipulado, que se vea obligado a buscar información sobre el tema planteado, y que escuche a sus compañeros.

Hoy en día existen múltiples herramientas que favorecen la interacción oral simultánea entre dos participantes (e.g. Skype, Messenger), o bien pensados para clases magistrales con escasa participación del alumno, como ocurre con plataformas de campus virtuales. Sin embargo, el uso de una herramienta de carácter asincrónico parece favorecer la participación equitativa de todos los estudiantes y que sus intervenciones puedan ser valoradas individualmente.

\section{EVALUACIÓN}

Las tareas de VOXOPOP se han evaluado como cualquier otro trabajo de clase que los alumnos tienen que elaborar semanalmente. Las tareas semanales contribuyen en un porcentaje elevado (35-40\%) a su nota final, ya que se tienen en cuenta el esfuerzo y la evolución en la producción escrita y oral que muestra el estudiante a lo largo del curso. El hecho de que la tarea sea parte de su puntuación ayuda a que se lleve a cabo con responsabilidad y que el alumno la vea como parte de su enseñanza-aprendizaje. A la hora de puntuar la intervención en el foro de voz se tienen en cuenta varios aspectos. Por una parte, se valora la adecuación a los requisitos de la tarea: (i) que los estudiantes hayan seguido las instrucciones especificadas para el ejercicio, como por ejemplo que no se hayan limitado a leer un texto escrito; (ii) que se hayan informado previamente sobre el tema propuesto; y (iii) que su respuesta se adecue a la tarea que se les ha planteado, ya sea 
manifestar una opinión o encontrar información concreta en una fuente. Por otra parte, se tienen en cuenta criterios lingüísticos: (i) fluidez; (ii) variedad de estructuras y vocabulario y (iii) corrección a nivel fonético, pragmático y sintáctico, teniendo esta última un menor peso global. De este modo, el alumno sabe que su intervención será evaluada de forma individual y que recibirá feedback por parte del profesor sobre los distintos parámetros y con especial énfasis en los errores que pueda cometer.

\section{CONCLUSIÓN}

A modo de conclusión cabe señalar la utilidad del programa VOXOPOP, y las posibilidades que tiene para un docente de lengua extranjera, ya que nos permite incrementar el tiempo de producción oral, que tantas veces se ve limitado en el aula, y que tan útil resulta para mejorar varias destrezas en una lengua extranjera y para consolidar contenidos. Las tareas que se pueden proponer variarán dependiendo del nivel del grupo de estudiantes. Gracias a muchas otras herramientas disponibles de forma gratuita en la web, podemos aumentar las áreas lingüísticas que el alumno tiene que trabajar para llevar a cabo una tarea en el foro de voz, insistiendo sobre todo en la práctica de producción y recepción oral.

\section{REFERENCIAS}

ARNAIZ CASTRO, P.; PEÑATE CABRERA, M. El papel de la producción oral (output) en el proceso de aprendizaje de una lengua extranjera (LE). Porta Linguarum: revista internacional de didáctica de las lenguas extranjeras, v. 1, p. 37-59, 2004.

BRACEY, B. ICT: A powerful new toll to teach literacy. In: BRACEY B.; CULVER, T. (Eds.). Harnessing the Potential of ICT for Education: A Multistakeholder Approach: Proceedings from the Dublin Global Forum of the United Nations ICT Task Force. New York: United Nations Publications, 2005. p. 137-140.

CONSEJO DE EUROPA, Marco común europeo de referencia para las lenguas: aprendizaje, enseñanza, evaluación. Traducción de Common European Framework of Reference for Languages: Learning, Teaching, Assessment. Strasbourg: Council of Europe, 2001. Madrid: MECD y Anaya, 2002.

EDIRISINGHA, P.; HAWKRIDGE, D.; FOTHERGILL, J. A Renaissance of Audio: Podcasting approaches for learning on campus and beyond. European Journal of Open Distance and E-

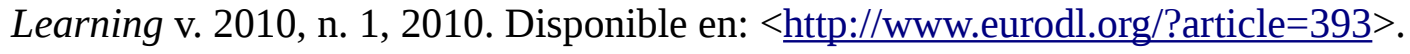

EVANS, T.D.; NATION, D.E. Dialogue in practice, research and theory in distance education. Open Learning, v. 4, n.2, p. 37-43, 1989.

FARRAND, C. M. Voxopop. Una manera fácil y divertida de practicar las destrezas orales fuera del aula. Koiné, Revista de l'Escola Oficial d`Idiomes de Castelló, v. 3, p. 22-25, 2011. 
Disponible

en:

$<$ http://www.eoicastello.es/eoi/upload/Activitats i serveis/Koine/Koine2011 v3.pdf>.

GÓMEZ MARTÍNEZ, S. Using web resources to support teachers and students with the teaching and practice of listening comprehension. Encuentro, v. 19, p. 20-31, 2010. Disponible

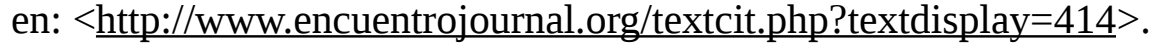

KOCH, P. Court records and cartoons: Reflections of spontaneous dialogue in early Romance texts. In: JUCKER, A. H., LEBSANFT, F.; FRITZ, G. (Eds.). Historical Dialogue Analysis. Amsterdam: John Benjamins Publishing Company, 1999. p. 399-429.

ÖSTERREICHER, W. Types of orality in text. In: BAKKER, E. J.; KAHANE, A. (Eds.). Written Voices, Spoke signs, Tradition, Performance, and the Epic Text. Harvard: Harvard University Press, 1997. p.190-214.

RICHARDSON, J. Literacy or the art of integration. In: AVIRAM, A.; RICHARDSON, J. (Eds.). Upon What Does the Turtle Stand?: Rethinking Education for the Digital Age. Dordrecht: Kluwer Academic Publishers, 2004. p. 135-149.

SHIPLEY, S.; MUILENBURG, L. How Do You Appropriately Assess English Language Learners? Integrating Voxopop Into Classroom Assessment. In: RESTA, P. (Ed.). Proceedings of Society for Information Technology \& Teacher Education International Conference 2012, 2012. p. 3764-3766.

VETTER, A.; CHANIER, T. Supporting oral production for professional purposes in synchronous communication with heterogeneous learners. Journal ReCALL v.18, n.1, p. 5-23, 2006. 
i VoiceThread ofrece diferentes versiones dependiendo del tipo de usuario final. Está disponible en la página $<$ http://www.voicethread.com>.

ii Ambos programas están disponibles en los correspondientes sitios web de Apache OpenOffice:

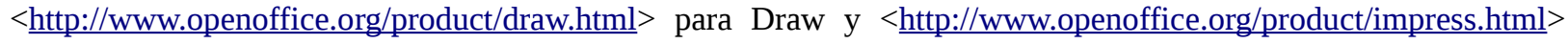
para Impress.

iii Free Dictionary ofrece una completa colección de diccionarios multilingües.

iv La herramienta "How Do You Say" está disponible en la página de Google Chrome: $<$ https://chrome.google.com/webstore/>. 